\title{
Variação sazonal dos grupos funcionais fitoplanctônicos em braços de um reservatório tropical de usos múltiplos no estado de São Paulo (Brasil)
}

Davi Gasparini Fernandes Cunha ${ }^{1,2}$ e Maria do Carmo Calijuri ${ }^{1}$

Recebido em 22/10/2010. Aceito em 26/09/2011

\begin{abstract}
RESUMO
(Variação sazonal dos grupos funcionais fitoplanctônicos em braços de um reservatório tropical de usos múltiplos no estado de São Paulo (Brasil)). A análise dos grupos funcionais fitoplanctônicos em sistemas aquáticos pode oferecer subsídios à conciliação dos usos múltiplos. Essa pesquisa investigou a variação sazonal desses grupos no reservatório de Itupararanga (SP). Quatro braços foram amostrados para determinação de temperatura da água, pH, Radiação Solar Fotossinteticamente Ativa Subaquática (RSFA), turbidez, fósforo total, nitrato e sólidos suspensos totais (SST), além da contagem e identificação do fitoplâncton e análise de dados meteorológicos. O estudo, realizado em agosto e outubro de 2009 e fevereiro de 2010, sugeriu variação sazonal dos grupos fitoplanctônicos $\left(\mathrm{p}^{*}<0,05\right)$, com predominância de organismos Sn, X1, M e F (inverno), X1, Y, F e K (primavera) e X1, J, K e W2 (verão). No inverno e na primavera, as principais variáveis que influenciaram a abundância dos grupos foram SST (7,4 a 48,1 $\left.\mathrm{mg} \mathrm{L}^{-1}\right)$ e turbidez (7 a $\left.96 \mathrm{UT}\right)$. No verão, foram determinantes $\mathrm{pH}(7,7$ a 9,8), temperatura $(27,8$ a 29 , $\left.1{ }^{\circ} \mathrm{C}\right)$ e RSFA $\left(>1.000 \mu \mathrm{E} \mathrm{m}^{-2} \mathrm{~s}^{-1}\right)$. Em face à significativa variabilidade ambiental dos braços do reservatório, pela influência dos rios tributários, e à predominância de grupos fitoplanctônicos característicos de ambientes eutróficos, recomenda-se seu contínuo monitoramento para evitar prejuízos aos usos do sistema aquático, sobretudo para abastecimento e recreação.
\end{abstract}

Palavras-chave: bacia do rio Sorocaba, comunidade fitoplanctônica, eutrofização, recursos hídricos, sistemas lênticos

\begin{abstract}
(Seasonal variation of phytoplankton functional groups in the arms of a tropical reservoir with multiple uses (SP, Brazil)). The assessment of phytoplankton functional groups may help water resource management, considering the multiple uses of this resource type. This research investigated the seasonal variation of these groups in the Itupararanga Reservoir (SP, Brazil). Four arms of the reservoir were sampled to determine the water temperature, $\mathrm{pH}$, underwater Photosynthetically Active Radiation (PAR), turbidity, total phosphorus, nitrate and total suspended solids (TSS), besides qualitative and quantitative aspects of the phytoplankton community and meteorological data. The study was carried out in August and October 2009, and in February 2010 , and suggested there is seasonal variation of phytoplankton groups $\left(\mathrm{p}^{*}<0.05\right)$, with the predominance of organisms Sn, X1, M and F (winter), X1, Y, F and K (spring) and X1, J, K and W2 (summer). The main variables that influenced the abundance of groups in the winter and in the spring were TSS (7.4 to $48.1 \mathrm{mg} \mathrm{L}^{-1}$ ) and turbidity ( 7 to $96 \mathrm{UT}$ ). Temperature $\left(27.8\right.$ to $29.1{ }^{\circ} \mathrm{C}$ ), $\mathrm{pH}$ (from 7.7 to 9.8 ) and PAR $\left(>1000 \mu \mathrm{E} \mathrm{m} \mathrm{m}^{-2} \mathrm{~s}^{-1}\right.$ ) were relevant in the summer. Considering the environmental variability of the arms of the reservoir (derived from the influence of tributaries), and the dominance of phytoplankton groups typical of eutrophic environments, we recommend continuous monitoring of this area so that different water uses can continue, particularly for supply and recreation.
\end{abstract}

Key words: Sorocaba River watershed, phytoplankton community, eutrophication, water resources, lentic systems

\footnotetext{
1 Universidade de São Paulo, Escola de Engenharia de São Carlos, São Carlos, SP, Brasil

2 Autor para correspondência: davig@sc.usp.br
} 


\section{Introdução}

Desde sua publicação original (Reynolds 1997), com posterior atualização (Reynolds et al. 2002), a classificação do fitoplâncton de acordo com os grupos funcionais desperta interesse da comunidade científica. Essa proposta de agrupamento das espécies apresenta vantagens em relação à classificação filogenética tradicional, visto que reúne os organismos com base em suas estratégias de sobrevivência e suas adaptações às condições ambientais, eliminando problemas relacionados a diferenças de acuracidade na identificação das espécies (Salmaso \& Padisák 2007). Esse esquema considera a tolerância ou a sensibilidade das espécies às variáveis físicas e químicas do sistema aquático por elas colonizado. Pode facilitar, ainda, a análise das interações entre o fitoplâncton e outras comunidades biológicas, como as macrófitas aquáticas (Fonseca \& Bicudo 2010). A classificação fitossociológica também permite a avaliação da influência das condições hidráulicas de um reservatório (e.g. tempo de residência), das características morfométricas e de uso e ocupação da bacia hidrográfica sobre as algas (Borges et al. 2008).

Pesquisas recentes empregaram a classificação dos grupos funcionais fitoplanctônicos e relacionaram a ocorrência e abundância desses agrupamentos às características ambientais de lagos e reservatórios. Baixas concentrações de nutrientes e elevada disponibilidade de luz estimularam o desenvolvimento dos grupos $\mathrm{Ze} \mathrm{M}$ em um lago oligotrófico tropical (Sarmento et al. 2006). Condições de estratificação ou mistura completa da coluna de água foram determinantes para favorecer diferentes agrupamentos em um lago eutrófico na Alemanha (Wilhelm \& Adrian 2008) e em três reservatórios australianos (Burford \& O’Donohue 2006). Diversos outros estudos investigaram a distribuição relativa dos grupos e os fatores abióticos e bióticos intervenientes (Huszar \& Caraco 1998, Salmaso 2002, Willén 2003, Padisák et al. 2006, Zhang et al. 2007, Dantas et al. 2008, Sarmento \& Descy 2008).

No Brasil, esforços se concentraram na avaliação da heterogeneidade espacial e da variabilidade temporal dos grupos funcionais em reservatórios para abastecimento de água (e.g. Bouvy et al. 2003), em reservatórios urbanos (Lopes et al. 2005, Fonseca \& Bicudo 2008), em lagos originados de planícies de inundação de rios (Huszar \& Reynolds 1997, Melo \& Huszar 2000, Bovo-Scomparin \& Train 2008) e em lagoas costeiras (Alves-de-Souza et al. 2006). Entretanto, esse tipo de abordagem é menos comum para reservatórios de usos múltiplos. Gemelgo et al. (2009) examinaram a variação sazonal dos grupos fitoplanctônicos nos reservatórios Billings e Guarapiranga (SP), cuja água é utilizada para abastecimento público, industrial e recreação. A principal conclusão nesse caso foi que a dinâmica temporal do fitoplâncton esteve associada à temperatura, extensão da zona eufótica, turbidez, condutividade, $\mathrm{pH}$ e concentrações de oxigênio dissolvido, nitrato e fósforo total.
A avaliação dos grupos funcionais fitoplanctônicos pode oferecer subsídios para o gerenciamento dos recursos hídricos. A ocorrência de alguns grupos está diretamente relacionada ao estado trófico do sistema aquático e, nesse sentido, a abordagem fitossociológica pode oferecer indicativos do grau de enriquecimento do lago ou reservatório estudado. Nesse contexto, o objetivo principal da presente pesquisa foi avaliar a variação sazonal dos grupos funcionais fitoplanctônicos em um reservatório tropical de usos múltiplos, o reservatório de Itupararanga (SP, Brasil). A principal motivação para esse estudo foi a relativa carência de informações sobre os grupos funcionais em sistemas aquáticos que apresentam usos diversos da água. Além disso, mais especificamente, a necessidade de apresentar subsídios para o manejo do ambiente estudado, cujo nível trófico tem aumentado nos últimos anos. Como objetivo específico, investigamos a influência das condições ambientais sobre a distribuição relativa desses agrupamentos fitoplanctônicos e analisamos quais foram os grupos mais bem sucedidos em face às características físicas e químicas da água nos diferentes períodos de coleta.

\section{Material e métodos}

O reservatório de Itupararanga está situado na bacia hidrográfica do rio Sorocaba (SP), que integra a Unidade de Gerenciamento de Recursos Hídricos número dez do estado de São Paulo (UGRHI 10). A área de influência da represa abrange os municípios de Alumínio, Cotia, Ibiúna, Mairinque, Piedade, São Roque, Vargem Grande Paulista e Votorantim (Fig. 1). Embora tenha sido construído em 1914 com o objetivo principal de produção de energia elétrica, atualmente se processam usos múltiplos no reservatório. Além da geração energética $\left(10,9 \mathrm{~m}^{3} \mathrm{~s}^{-1}, 55 \mathrm{~mW}\right)$, o sistema é utilizado para abastecimento público $\left(2,2 \mathrm{~m}^{3} \mathrm{~s}^{-1}\right)$, irrigação de propriedades agrícolas $\left(0,13 \mathrm{~m}^{3} \mathrm{~s}^{-1}\right)$, navegação e lazer.

O tempo de residência da água no reservatório normalmente varia entre 4 e 13 meses, em consonância com as flutuações hidrológicas naturais ao longo do ano. Possui capacidade de armazenamento de 286 milhões $\mathrm{de}^{3}$ e as formas principais de uso do solo em sua área de drenagem são agricultura (42\%) e vegetação nativa (25\%), de acordo com Garcia et al. (1999). Relatórios da CETESB (Companhia Ambiental do Estado de São Paulo) descreveram o contínuo incremento do grau de trofia do reservatório de Itupararanga e o aumento da participação relativa das Cianobactérias na comunidade fitoplanctônica e da velocidade de degradação da qualidade da água nos anos recentes (CETESB 2007, 2008, 2009).

Quatro estações de amostragem foram escolhidas em braços do reservatório (Fig. 1, Tab. 1) para coleta de água e estudo da comunidade fitoplanctônica em escala sazonal. Essas estações se localizam na margem esquerda da represa, que concentra a maior parte das interferências antrópicas (e.g. construções, novos loteamentos de casas e áreas 

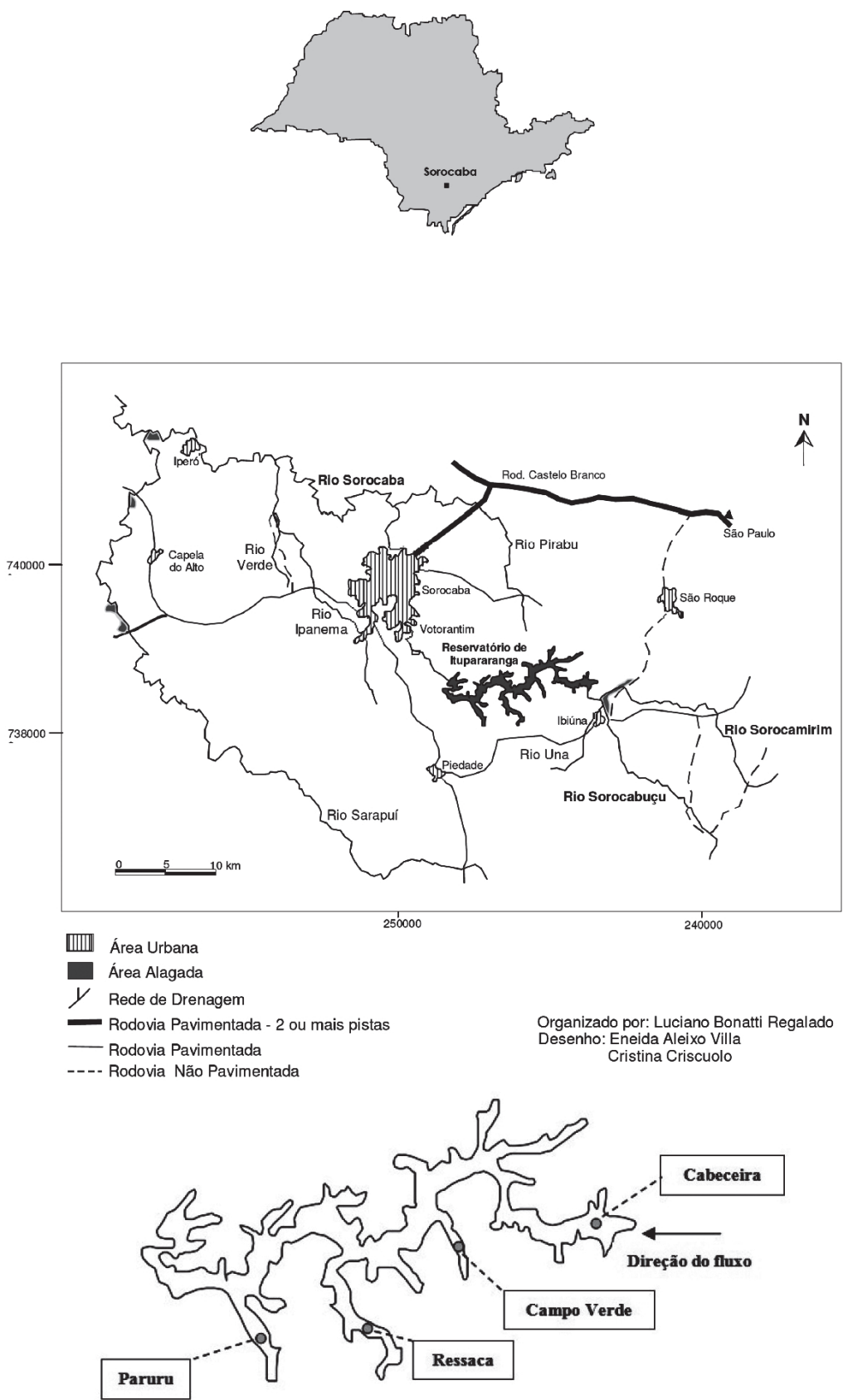

Figura 1. Localização da área de estudo no estado de São Paulo, Brasil, e cidades mais importantes próximas ao reservatório de Itupararanga. Localização aproximada das quatro estações de coleta no sistema aquático (Cabeceira do reservatório, Ressaca, Paruru e Campo Verde). Adaptado de Marciano (2001)

com cultivos agrícolas). Foram efetuadas três coletas para abranger período de inverno (agosto de 2009), primavera (outubro de 2009) e verão (fevereiro de 2010). Dados climatológicos (precipitação pluviométrica, temperatura do ar e velocidade dos ventos) foram obtidos de publicações do Instituto Nacional de Meteorologia (INMET 2009, 2010), a partir de uma estação meteorológica em Sorocaba-SP (Latitude: $23,48^{\circ} \mathrm{S}$; Longitude: $47,43^{\circ} \mathrm{W}$; Altitude: $645 \mathrm{~m}$ ).

Amostras subsuperficiais de água foram coletadas, armazenadas em frascos plásticos e congeladas para posterior análise. Foram determinadas, em laboratório, as concentrações de fósforo total (PT), nitrato $\left(\mathrm{NO}_{3}\right)$ e sólidos suspensos totais (SST) por meio dos métodos propostos por APHA (2005). Foram também obtidos alguns dados in situ, como temperatura da água (T) e pH (Sonda Multiparâmetros Yellow Springer 556 ), Radiação Solar Fotossinteticamente Ativa Subaquática (RSFA, medida em subsuperfície com auxílio de Radiômetro Quanta-Meter Ly-cor LI- $1.400^{\circ}$ ) e turbidez (turbidímetro Marconi ${ }^{\oplus}$. 
Tabela 1. Coordenadas geográficas, profundidade média $(\mathrm{m})$ e descrição das quatro estações de coleta no reservatório de Itupararanga.

\begin{tabular}{lccc}
\hline Estação de coleta & Coordenadas geográficas & $\begin{array}{c}\text { Profundidade média } \\
(\mathrm{m})\end{array}$ & Descrição \\
\hline Cabeceira do reservatório & $23^{\circ} 37^{\prime} 28.30^{\prime \prime} \mathrm{S}$ & 7,1 & $\begin{array}{c}\text { Entrada dos principais rios formadores do reservatório, rios } \\
\text { Sorocabuçu e Sorocamirim }\end{array}$ \\
& $47^{\circ} 13^{\prime} 52.24^{\prime \prime} \mathrm{W}$ & 2,0 & Entrada do Córrego da Ressaca \\
& $23^{\circ} 39^{\prime} 24.65^{\prime \prime} \mathrm{S}$ & & Entrada do Córrego do Paruru \\
& $47^{\circ} 18^{\prime} 36.50^{\prime \prime} \mathrm{W}$ & 1,6 & \\
Ressaca & $23^{\circ} 40^{\prime} 10.72^{\prime \prime} \mathrm{S}$ & & Entrada do Córrego do Campo Verde \\
\hline
\end{tabular}

Em relação à comunidade fitoplanctônica, amostras de água foram fixadas com lugol acético e os organismos foram identificados a partir de microscópio óptico invertido Olympus $\mathrm{CK}^{\circ}$. Para isso, foram utilizadas chaves de identificação taxonômica disponíveis na literatura, tais como Anagnostidis \& Komarék (1986, 1988, 1989), Bicudo \& Menezes (2005), Bourrely (1972a, 1972b, 1981), Castro et al. (1991), Komarék \& Fott (1983), Parra et al. (1980, 1982, 1983) e Sant'anna et al. (2006). Além da densidade fitoplanctônica total, calculada a partir das recomendações de APHA (2005), foi efetuado o agrupamento funcional com base na classificação fitossociológica proposta por Reynolds et al. (2002), com os cuidados sugeridos por Padisák et al. (2009).

Análises estatísticas foram processadas para verificar a significância da componente sazonal sobre os resultados e para facilitar a visualização dos fatores ambientais que estiveram relacionados à predominância de determinados grupos funcionais fitoplanctônicos. Assim, com auxílio do software Systat $12^{\circ}$, os dados foram submetidos à Análise de Variância Multivariada (MANOVA), com nível de confiança de $95 \%\left(\mathrm{p}^{*}<0,05\right)$ e a Análise de Componentes Principais (PCA) a partir de dois eixos.

\section{Resultados e discussão}

A precipitação pluviométrica mensal em agosto e outubro de 2009 e fevereiro de 2010 foi de $43 \mathrm{~mm}, 96$ $\mathrm{mm}$ e $88 \mathrm{~mm}$, respectivamente (Fig. 2A). Ao contrário do esperado para a época, julho de 2009 foi um mês atipicamente chuvoso (aproximadamente $200 \mathrm{~mm}$ ). Assim, a influência das chuvas do mês de julho faz com que agosto de 2009, embora com precipitação pluviométrica total de apenas $43 \mathrm{~mm}$, não possa ser considerado como mês típico de estiagem. O mês de fevereiro de 2010, por sua vez, apresentou precipitação inferior ao esperado. Entretanto, esse mês foi considerado como abrangente de período úmido pela influência dos meses chuvosos precedentes (novembro - $226 \mathrm{~mm}$, dezembro - $288 \mathrm{~mm}$ e janeiro - 360 $\mathrm{mm})$. Somente na semana anterior à coleta de outubro, houve precipitação de $49 \mathrm{~mm}$, superior ao precipitado nas semanas anteriores às amostragens de agosto $(0 \mathrm{~mm}) \mathrm{e}$ fevereiro $(11 \mathrm{~mm})$. A temperatura do ar oscilou, ao longo de 2009 e 2010, de acordo com as estações do ano, com média máxima de $24,5{ }^{\circ} \mathrm{C}$ em fevereiro de 2010 (Fig. 2B). Em relação à velocidade dos ventos, destaque para a coleta da primavera (média no mês igual a $2,7 \mathrm{~m} \mathrm{~s}^{-1} \mathrm{e}$ média no dia da coleta de 2,9 $\left.\mathrm{m} \mathrm{s}^{-1}\right)$. Em fevereiro, por outro lado, foram registradas velocidades menores, com média mensal de $1,7 \mathrm{~m} \mathrm{~s}^{-1}$ e inferior a $1,0 \mathrm{~m} \mathrm{~s}^{-1}$ especificamente no dia da amostragem (Fig. 2C).

Em função das profundidades relativamente pequenas das estações amostrais (Tab. 1) e da turbulência gerada pela entrada dos tributários, a coluna de água sempre apresentou mistura completa nos locais avaliados. No verão, foram observadas, além das maiores temperaturas de água (média de $28,7^{\circ} \mathrm{C}$ nas quatro estações de coleta) e elevadas RSFA, os maiores valores de $\mathrm{pH}$ (média acima de 8,0 ) e das densidades fitoplanctônicas, que variaram entre $3,8610^{3} \mathrm{e}$ $20,9110^{3}$ ind $\mathrm{mL}^{-1}$ (Fig. 3). O caráter mais alcalino da água é compatível com as maiores densidades do fitoplâncton e, presumidamente, com a maior taxa fotossintética e produção primária (Fig. 3B e $3 \mathrm{H}$ ). A variação sazonal do $\mathrm{pH}$ pode ser explicada pelo aumento do consumo de $\mathrm{CO}_{2}$ durante o verão (Palma et al. 2010). Na época de transição (primavera), por outro lado, as estações de coleta no reservatório de Itupararanga apresentaram as máximas concentrações de fósforo total, nitrato, SST e picos de turbidez (96 UT). As densidades fitoplanctônicas nesse período foram mais baixas e flutuaram entre 0,55 e $8,4310^{3}$ ind $\mathrm{mL}^{-1}$, o que está associado, possivelmente, à menor transparência da água (Fig. 3F e 3G). Não se deve descartar, também, a maior velocidade dos ventos no mês de outubro (Fig. 2C). Calijuri et al. (2002) destacaram, dentre outros fatores, a ação combinada do vento e da chuva como fator regulador da estrutura da comunidade fitoplanctônica em escala sazonal em um reservatório tropical. Assim, a influência dos padrões climáticos em menor escala (i.e. diária, na semana anterior às coletas) foi significativa. Em outubro, a lâmina precipitada na semana precedente à amostragem (Fig. 2A) alterou as características da coluna de água e, consequentemente, afetou a comunidade fitoplanctônica. 

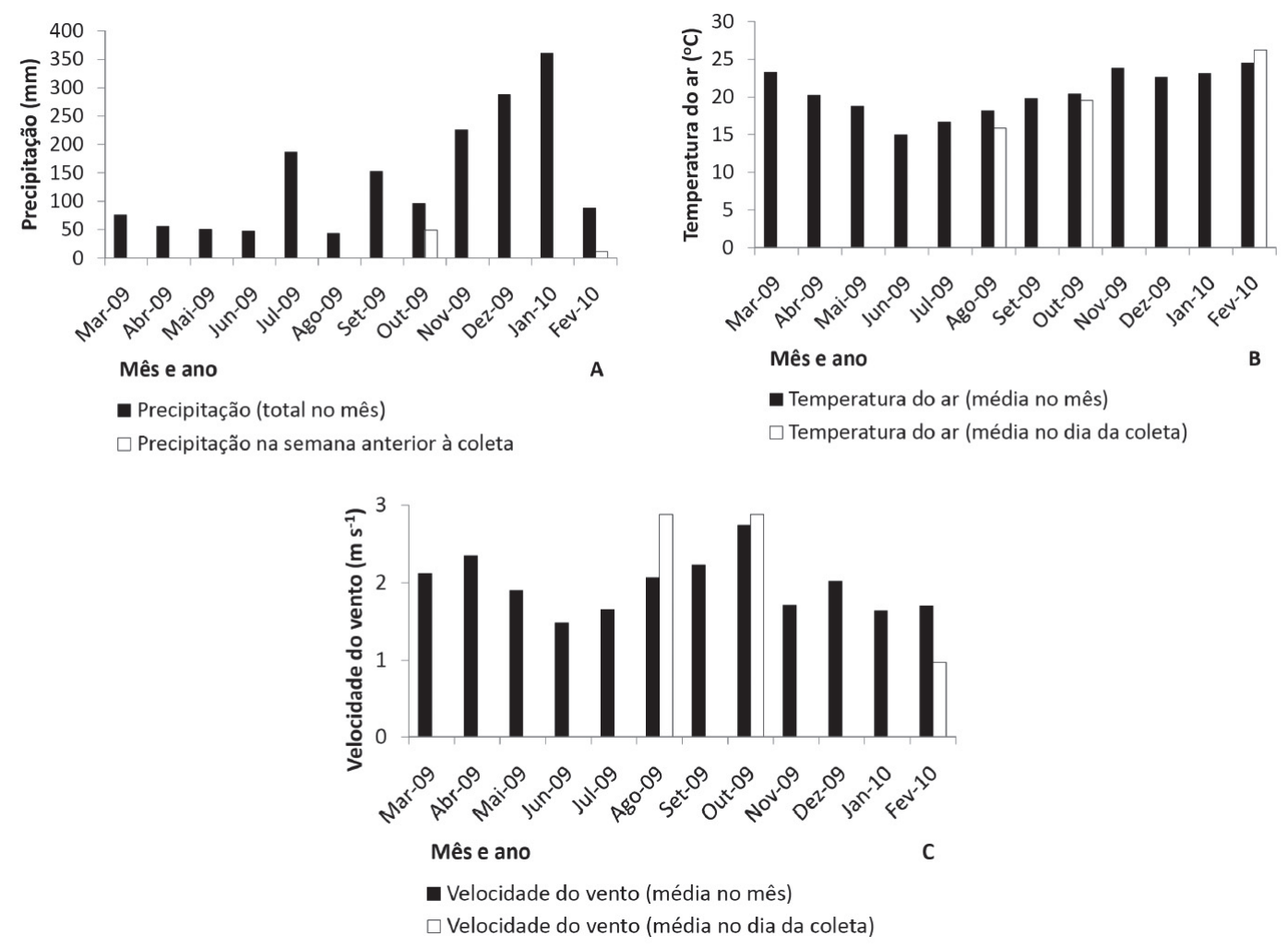

Figura 2. Dados climatológicos da área de estudo nos anos de 2009 e 2010: precipitação pluviométrica (A), temperatura do ar (B) e velocidade do vento (C). Para cada variável, são apresentados os totais ou as médias referentes ao mês e aos dias de coleta ou à semana antecedente.

Em comparação a outros reservatórios brasileiros, as densidades observadas no reservatório de Itupararanga se coadunam com aquelas encontradas em ambientes mesoeutróficos. Silva et al. (2005) relataram uma densidade média de $1,210^{3}$ ind $\mathrm{mL}^{-1}$ no reservatório Foz do Areia (PR), eutrófico, e Fonseca \& Bicudo (2008) não encontraram densidades fitoplanctônicas superiores a $12,210^{3}$ ind $\mathrm{mL}^{-1}$ no Lago das Garças (SP), hipereutrófico. No caso do reservatório Mundaú $(\mathrm{PE})$, houve predomínio de cianobactérias e as densidades totais estiveram entre 3,62 e 18,2 10 ${ }^{3}$ ind $\mathrm{mL}^{-1}$ (Moura et al. 2007). O reservatório de Barra Bonita (SP) apresentou densidades fitoplanctônicas da ordem de $10^{6}$ ind $\mathrm{mL}^{-1}$, caracterizando-o como eutrófico (Calijuri et al. 2002).

A componente sazonal foi considerada estatisticamente significativa $\left(\mathrm{p}^{*}<0,05\right)$, o que ressalta a importância da variação temporal da qualidade da água no reservatório de Itupararanga. O ordenamento gerado pela Análise de Componentes Principais sugeriu que sete variáveis contribuíram para a variação dos dados ao longo do período analisado (Tab. 2). O Fator 1 foi responsável pela explicação de $70 \%$ da variância e o Fator 2, por 15\%. A análise mostrou o efeito antagônico das variáveis com carga positiva $(\mathrm{pH}$, RSFA, temperatura e densidade fitoplanctônica) versus as de carga negativa (turbidez, nitrato e SST). Isso sugere que a limitação por luz foi preponderante nas quatro estações
Tabela 2. Porcentagem total da variância explicada pelos componentes principais (Fator 1 e Fator 2) e as correlações entre esses fatores e as variáveis da água.

\begin{tabular}{lcc}
\hline \multirow{2}{*}{ Variância explicada } & Fator 1 & Fator 2 \\
\cline { 2 - 3 } & $70,3 \%$ & $15,4 \%$ \\
\hline $\mathrm{pH}$ & 0,894 & 0,347 \\
Turbidez & $-0,874$ & 0,243 \\
RSFA & 0,850 & 0,461 \\
Temperatura & 0,835 & 0,487 \\
$\mathrm{NO}_{3}$ & $-0,810$ & 0,442 \\
$\mathrm{SST}$ & $-0,810$ & 0,219 \\
Densidade fitoplanctônica & 0,791 & $-0,456$ \\
\hline
\end{tabular}

de coleta, visto que as variáveis turbidez e SST foram ordenadas em oposição à densidade fitoplanctônica, situação semelhante à observada para o reservatório do Castanhão, CE (Molisani et al. 2010). Esses pesquisadores relataram a influência fluvial sobre as concentrações de material em suspensão nesse reservatório, o que reduziu a produção primária fitoplanctônica.

A PCA não considerou relevantes as concentrações de fósforo total para a explicação dos resultados. Isso pode ser interpretado como uma indicação de que, provavelmente, a comunidade fitoplanctônica não esteve limitada por esse 

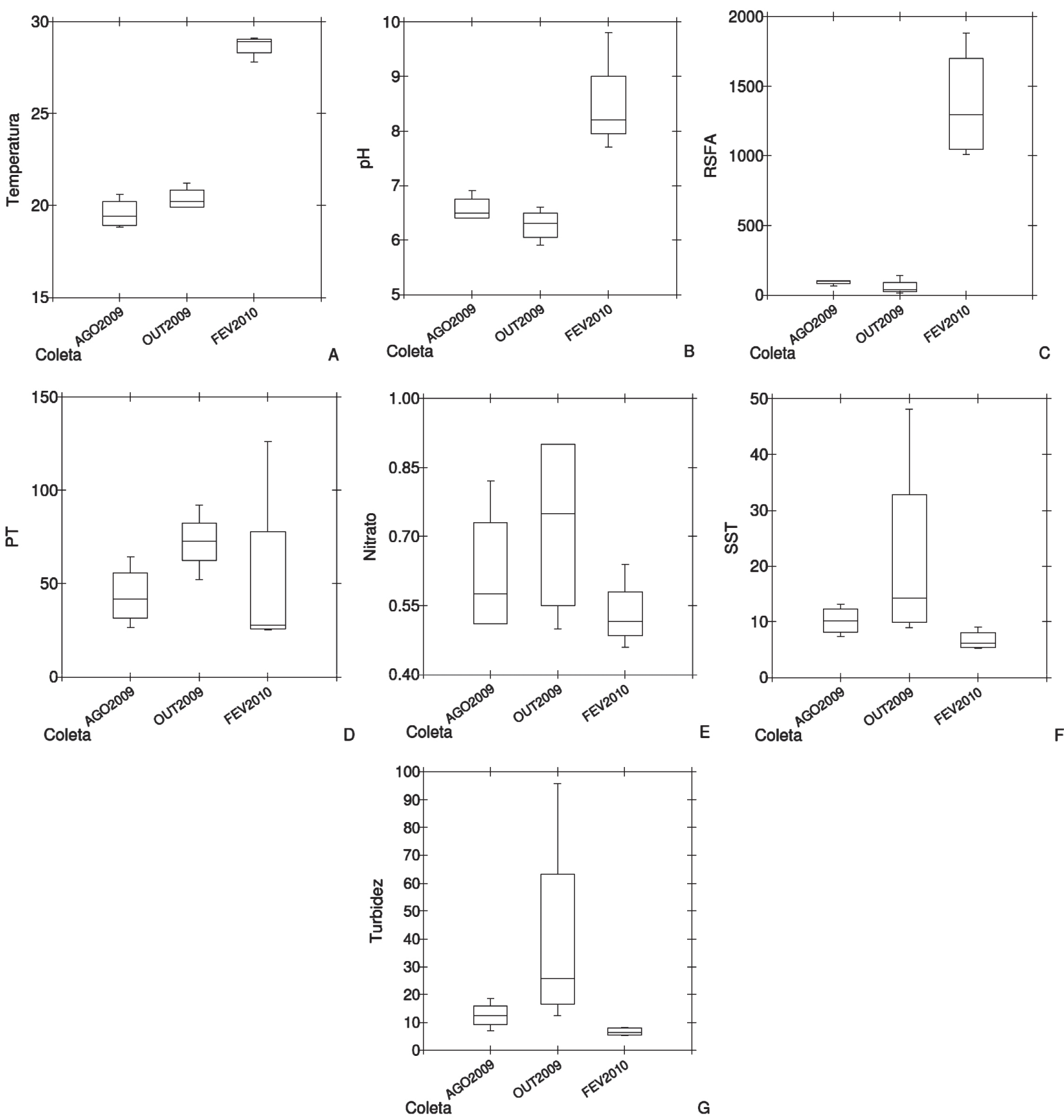

Figura 3. Boxplots das variáveis da água estudadas no reservatório de Itupararanga nas coletas de agosto e outubro de 2009 e fevereiro de 2010 : temperatura (A), $\mathrm{pH}(\mathrm{B})$, radiação solar fotossinteticamente ativa em subsuperfície (C), fósforo total (D), nitrato (E), sólidos suspensos totais (F), turbidez (G) e densidade total fitoplanctônica $(\mathrm{H})$.

nutriente em nenhum dos períodos analisados. $\mathrm{O}$ aporte de fósforo proveniente dos córregos que deságuam em cada um dos braços estudados e a concentração média de fósforo nas estações de amostragem $\left(56 \mu \mathrm{g} \mathrm{L}^{-1}\right)$ também apontam para essa mesma conclusão. Concentrações de fósforo total acima de $52 \mu \mathrm{g} \mathrm{L}^{-1}$ caracterizam reservatórios tropicais como eutróficos (Lamparelli 2004).

Moura et al. (2007) relataram limitação por nitrogênio no reservatório de Mundaú (PE, Brasil) em função das elevadas concentrações de fósforo disponíveis na água (médias entre 75 e $105 \mu \mathrm{g} \mathrm{L}^{-1}$ ). Entretanto, no caso dos braços avaliados no reservatório de Itupararanga, provavelmente não ocorre limitação por nitrogênio, uma vez que as concentrações de nitrato resultaram sempre superiores a $0,45 \mathrm{mg} \mathrm{L}^{-1}$. A limitação por nitrogênio ocorre quando a concentração de nitrato é inferior a $10 \mu \mathrm{g} \mathrm{L}^{-1}$ (Haande et al. 2010). Além disso, não deve ser negligenciada a capacidade das cianobactérias de fixar o nitrogênio atmosférico 
por meio dos heterocistos (Fiore et al. 2005, Figueredo \& Giani 2009).

Foram encontrados 74 táxons fitoplanctônicos nas amostras do reservatório, pertencentes a oito classes taxonômicas, de acordo com a seguinte distribuição: Chlorophyceae (40,5\%), Cyanobacteria (18,9\%), Bacillariophyceae $(12,2 \%)$, Euglenophyceae $(12,2 \%)$, Chrysophyceae $(5,4 \%)$, Cryptophyceae $(5,4 \%)$, Chlamydophyceae $(4,1 \%)$ e Dynophyceae $(1,4 \%)$. As espécies que apresentaram as maiores abundâncias relativas foram: Botryococcus braunii, Cylindrospermopsis raciborskii, Kirchneriella obesa, Microcystis aeruginosa, Monoraphidium contortum e Pseudokephyrion tatricum (inverno); Aphanocapsa elachista, Chlorella vulgaris, Cryptomonas erosa, Microcystis aeruginosa e Monoraphidium contortum (primavera); Ankistrodesmus mirabilis, Monoraphidium contortum, Monoraphidium irregulare e Trachelomonas intermedia (verão).

Nos três períodos amostrais, foram observados 23 grupos funcionais fitoplanctônicos (A, C, D, N, P, T, S1, Sn, Z, X2, X1, Y, E, F, G, J, K, H1, Lo, M, MP, W1 e W2). Trata-se de uma diversidade elevada de agrupamentos quando comparada a outros ambientes aquáticos (e.g. oito grupos descritos por Alves-de-Souza et al. 2006 na Lagoa Comprida-RJ; 18 grupos observados por Moura et al. 2007 no reservatório Mundaú-PE; 13 grupos relatados por Fonseca \& Bicudo 2008 no Lago das Garças-SP). Essa diversidade significativa pode ser associada à instabilidade da coluna de água das estações amostradas, que se situam na desemboca- dura de córregos e, portanto, estão submetidas a variações drásticas em relação à qualidade da água e ao escoamento. Esse sistema sujeito a diferentes condições ambientais não permitiu a dominância de uma única espécie fitoplanctônica por longo período de tempo. Nas diferentes fases do ciclo hidrológico, quatro a seis espécies apresentaram maior abundância relativa, mas nunca com contribuição acima de $50 \%$ do total. Caso semelhante foi relatado por Melo \& Huszar (2000) para o lago da Batata (AM), ambiente que apresentou marcante dinâmica sazonal fitoplanctônica, a qual foi influenciada principalmente pela flutuação do nível hidrométrico na respectiva planície de inundação.

A contribuição relativa dos grupos fitoplanctônicos apresentou variação temporal relevante do ponto de vista estatístico $\left(\mathrm{p}^{*}<0,05\right)$. Os quatro agrupamentos mais frequentes em cada coleta foram (Fig. 4): Sn, X1, M e F (agosto), X1, Y, F e K (outubro) e X1, J, Ke W2 (fevereiro). Tais grupos são típicos, em sua maioria, de ambientes quentes, rasos, meso-eutróficos e com coluna de água misturada (Reynolds et al. 2002).

A participação dos grupos Sn e M foi relevante apenas em agosto de 2009 (máximos de 36,2\% na estação Ressaca e $12,4 \%$ na estação Cabeceira, respectivamente), com porcentagens não significativas em outubro de 2009 e fevereiro de 2010. Isso pode estar associado à susceptibilidade desses grupos ao aumento da vazão (flushing) que ocorre nos períodos de maior precipitação (Reynolds et al. 2002). Além disso, a participação das algas pertencentes aos códons $Y$ e F foi maior em agosto e outubro de 2009. Tais grupos
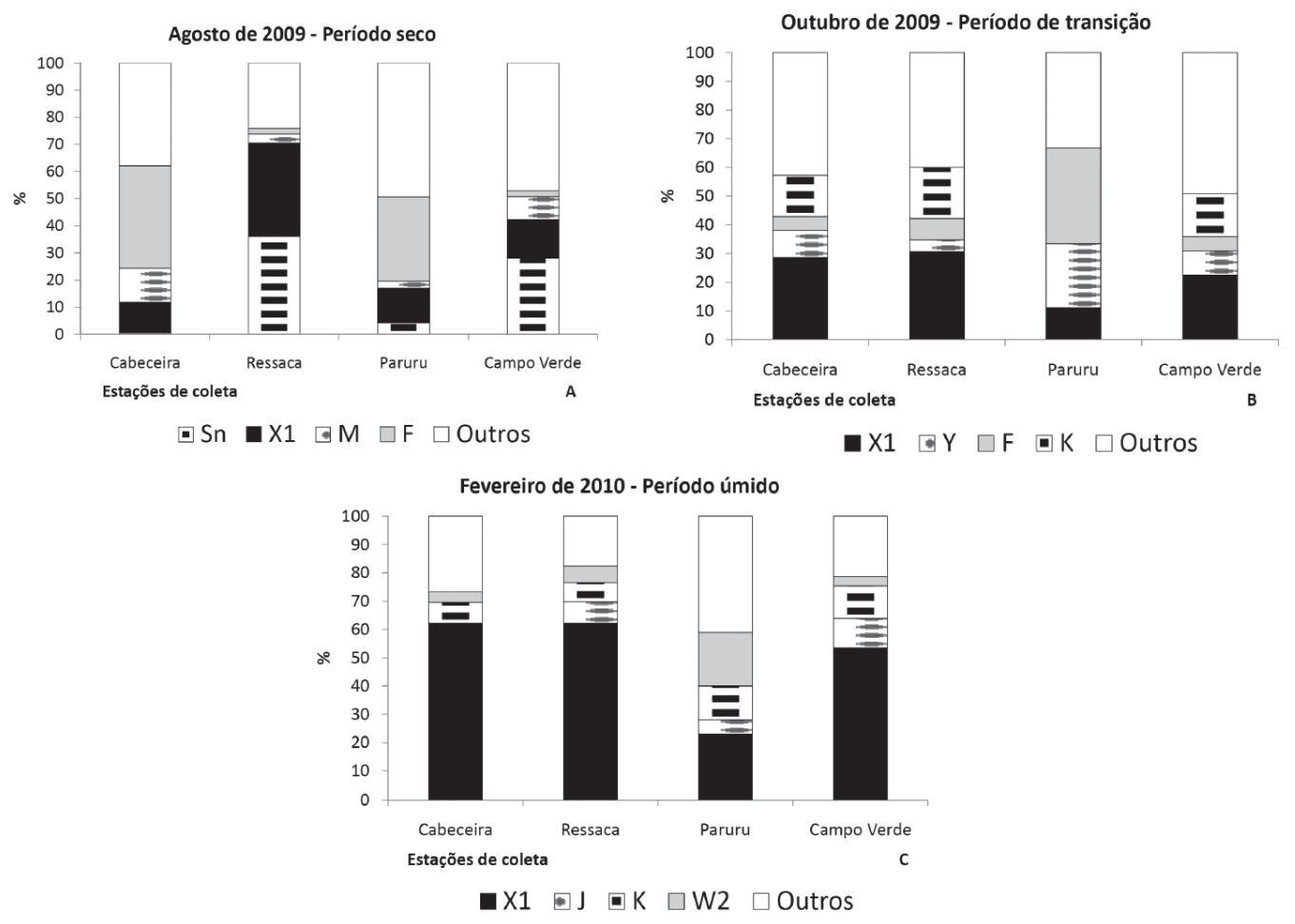

Figura 4. Principais grupos fitoplanctônicos e sua abundância relativa no reservatório de Itupararanga em agosto (A) e outubro (B) de 2009 e fevereiro (C) de 2010, de acordo com a classificação fitossociológica de Reynolds. 


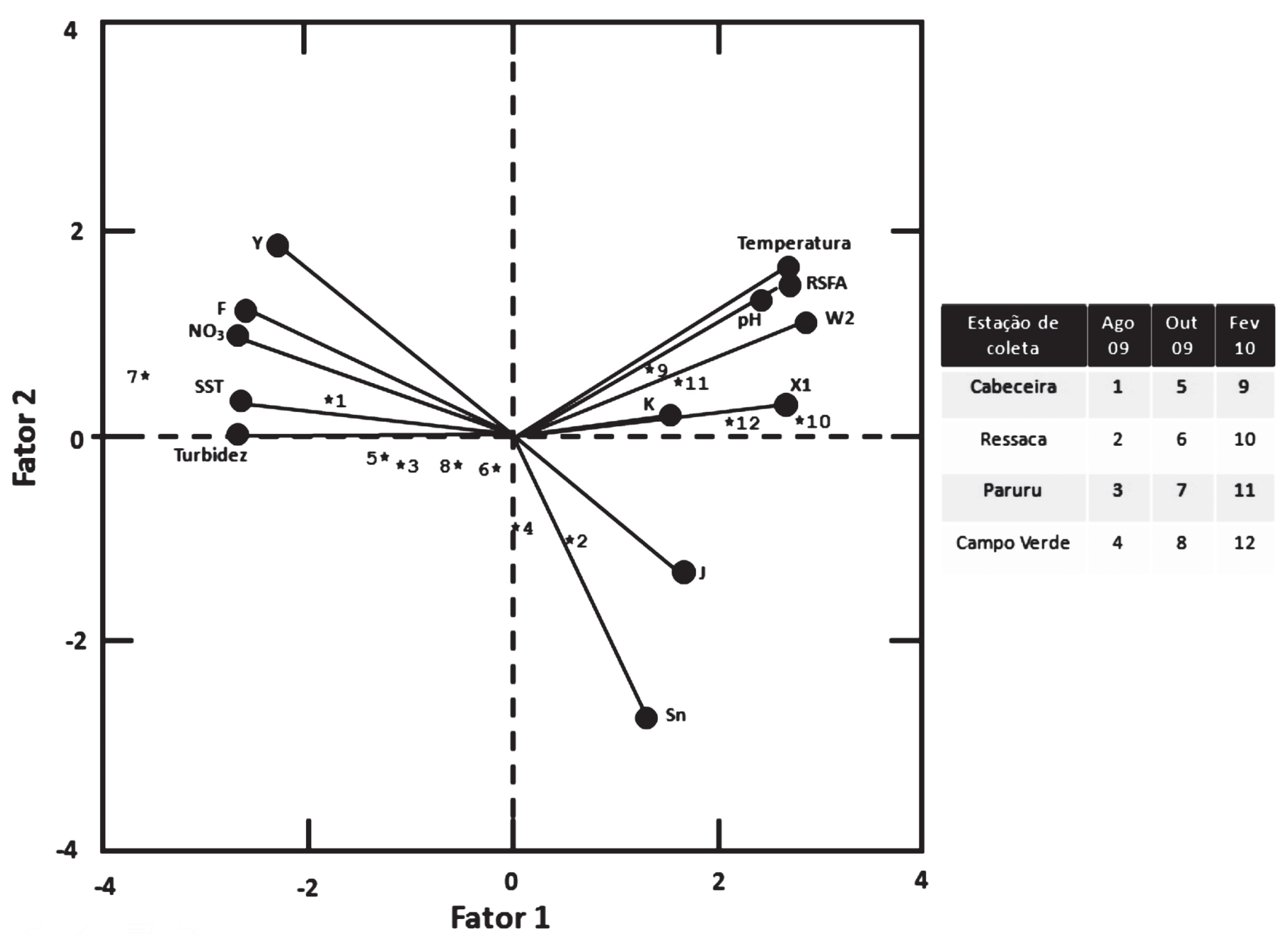

Figura 5. Biplot dos componentes principais (Fatores 1 e 2). Os vetores plotados indicam os scores de correlação obtidos entre as variáveis da água (características físicas e químicas e grupos funcionais fitoplanctônicos) e os dois fatores. Os números no interior dos quadrantes se referem às estações amostrais no inverno ( 1 a 4), primavera ( 5 a 8 ) e verão ( 9 a 12 ).

são tolerantes à baixa disponibilidade de luz (que ocorreu justamente nesses meses - Fig. 3C) e a elevados valores de turbidez da água, também observados nessas duas coletas, principalmente em outubro (Fig. 3G). Já no verão, houve mudança na estrutura da comunidade e outros organismos foram favorecidos pela maior disponibilidade de RSFA (códon J, com máximo de 12,0\% na estação Paruru). Nesse período, também mereceram destaque o grupo X1 (23,0\% a $62,2 \%)$, que se refere ao nanoplâncton de ambientes eutróficos, e o grupo W2 (3,3\% a 19,0\%), característico de ambientes mesotróficos.

As principais variáveis ambientais que influenciaram a abundância dos grupos fitoplanctônicos foram SST, turbidez $\mathrm{e} \mathrm{NO}_{3}$ no inverno e na primavera e $\mathrm{pH}$, temperatura e RSFA no verão (Fig. 5). O biplot também confirmou a flexibilidade dos grupos Y e F frente às condições de menor iluminação, o que lhes garantiu vantagem adaptativa nas estações seca e intermediária. Esses grupos foram mais bem sucedidos nesses períodos por estarem aptos a tolerar maiores valores de turbidez da água e maiores concentrações de SST. Esses resultados corroboram o estudo de Nabout et al. (2006), que verificaram a predominância do grupo $\mathrm{Y}$ em lagos do rio Araguaia (GO/
MT) no período de estiagem e ressaltaram a sua ocorrência, sobretudo das Cryptophyceae, em ambientes que apresentam mistura total da coluna de água. O gênero Cryptomonas, especificamente, caracteriza-se por taxas significativas de absorção de fósforo, o que lhes garante crescimento relativamente rápido (Huszar et al. 2000, Albay \& Akçaalan 2003).

No verão, as menores concentrações de nutrientes como fósforo total e nitrato (Fig. 3D e 3E), aliadas à maior disponibilidade de luz, permitiram a gênese de condições favoráveis a algas pertencentes aos códons J e W2. No entanto, houve domínio do grupo $\mathrm{X} 1$, representado principalmente pelos gêneros nanoplanctônicos Monoraphidium e Ankistrodesmus. Isso confirma os resutados da pesquisa realizada por Rangel et al. (2009), que observaram, para o lago Monte Alegre (SP), maior contribuição do grupo X1 no verão. Os pesquisadores atribuíram as menores porcentagens de organismos X1 no inverno ao grazing pelo zooplâncton, em função da presença significativa de organismos filtradores nesse período. A turbulência verificada na estação chuvosa, como resultado da maior vazão dos rios tributários, também pode ter favorecido esse grupo, como verificado por outros autores (Happey-Wood 1988, Moura et al. 2007). 
Os resultados obtidos permitem concluir que os braços do reservatório de Itupararanga apresentaram uma diversidade significativa de grupos funcionais, com presença marcante de agrupamentos típicos de ambientes eutrofizados. Indicam, portanto, a necessidade de manejo do reservatório com vistas ao controle do enriquecimento por nutrientes pela influência de seus rios formadores. Houve variação sazonal significativa da abundância relativa dos grupos nas estações amostradas, o que foi atribuído, principalmente, à dinâmica temporal das características físicas e químicas da água, além da influência dos fatores climáticos, como vento e precipitação pluviométrica. Cabe ressaltar, no entanto, que o período avaliado foi relativamente atípico do ponto de vista de distribuição de chuvas, sendo necessária cautela na análise dos resultados.

Em função de sua inerente instabilidade, os braços estão mais susceptíveis às mudanças de uso e ocupação na bacia hidrográfica e, como consequência, mais vulneráveis à degradação da qualidade da água pelo impacto negativo de seus tributários. A rápida resposta da qualidade da água dos braços a eventos de precipitação pluviométrica na bacia hidrográfica, como verificado em outubro, foi observada nessa pesquisa. A diversidade de condições favoreceu, em alguns períodos, a colonização por espécies fitoplanctônicas potencialmente tóxicas, como por exemplo as do grupo Sn e M. As implicações da variação temporal dos grupos do fitoplâncton nos braços do sistema aquático estudado, portanto, estendem-se aos usos múltiplos do reservatório, sobretudo aos usos mais nobres (abastecimento e recreação), e devem ser alvo de acompanhamento contínuo.

\section{Agradecimentos}

Os autores gostariam de expressar sinceros agradecimentos à FAPESP (Fundação de Amparo à Pesquisa do Estado de São Paulo) pela bolsa de doutorado direto concedida a DGF. Cunha (Processo 2009/50842-2) e pelo auxílio financeiro, na forma de projeto temático, à MC. Calijuri (Processo 2008/55636-9). Dois revisores anônimos avaliaram o manuscrito e forneceram sugestões valiosas para aprimorar o texto.

\section{Referências bibliográficas}

Albay, M. \& Akçaalan, R. 2003. Factors influencing the phytoplankton steady state assemblages in a drinking-water reservoir (Ömerli reservoir, Istanbul). Hydrobiologia 502(1-3): 85-95.

Alves-de-Souza, C.; Menezes, M. \& Huszar, V.L.M. 2006. Phytoplankton composition and functional groups in a tropical humic coastal lagoon, Brazil. Acta Botanica Brasilica 20(3): 701-708.

Anagnostidis, K. \& Komarék, J. 1986. Modern approach to the classification system of Cyanophytes. 2 - Chloroococales. Algological Studies 73: $157-226$.

Anagnostidis, K. \& Komarék, J. 1988. Modern approach to the classification system of Cyanophytes. 3 - Oscillatoriales. Algological Studies 50-53: 327-472.
Anagnostidis, K. \& Komarék, J. 1989. Modern approach to the classification system of Cyanophytes. 4 - Nostocales. Algological Studies 82: 247-345.

APHA - American Public Health Association. 2005. Standard Methods for the examination of water and wastewater. $21^{\text {st }} \mathrm{ed}$. Washington, APHA.

Bicudo, C.E.M. \& Menezes, M. 2005. Gêneros de algas de águas continentais do Brasil - chave para identificação e descrições. São Carlos, RiMa.

Borges, P.A.F.; Train, S. \& Rodrigues, L.C. 2008. Spatial and temporal variation of phytoplankton in two subtropical Brazilian reservoirs. Hydrobiologia 607(1): 63-74.

Bourrely, P. 1972a. Les algues d'eau douce - initiation à la systématique - les algues vertes. Paris, N. Boubee.

Bourrely, P. 1972b. Les algues d'eau douce - les algues bleues et rouges - les eugléniens, péridiniens et cryptomonadines. Paris, N. Boubee.

Bourrely, P. 1981. Les algues d'eau douce - les algues jaunes et brunes. Paris, N. Boubee.

Bouvy, M.; Nascimento, S.M.; Molica, R.J.R.; Ferreira, A.; Huszar, V.L.M. \& Azevedo, S.M.F.O. 2003. Limnological features in Tapacurá reservoir (northeast Brazil) during a severe drought. Hydrobiologia 493(1-3): 115-130.

Bovo-Scomparin, V.M. \& Train, S. 2008. Long-term variability of the phytoplankton community in an isolated floodplain lake of the Ivinhema River State Park, Brazil. Hydrobiologia 610(1): 331-344.

Burford, M.A. \& O’Donohue, M. 2006. A comparison of phytoplankton community assemblages in artificially and naturally mixed subtropical water reservoirs. Freshwater Biology 51(5): 973-982.

Calijuri, M.C.; Dos Santos, A.C.A \& Jati, S. 2002. Temporal changes in the phytoplankton community structure in a tropical and eutrophic reservoir (Barra Bonita, S.P.-Brazil). Journal of Plankton Research 24(7): 617-634.

Castro, A.A.J.; Bicudo, C.E.M. \& Bicudo, D.C. 1991. Criptógamos do Parque Estadual das Fontes do Ipiranga, São Paulo, SP. Algas: $2-$ Chryptophyceae. Hoehnea 18: 87-106.

CETESB - Companhia de Tecnologia de Saneamento Ambiental. 2007, 2008, 2009. Relatórios de Qualidade das Águas Interiores do Estado de São Paulo. Disponível em: http://www.cetesb.gov.br/agua/rios/ publicacoes.asp (Acesso em 15/07/2010).

Dantas, E.W.; Moura, A.N.; Bittencourt-Oliveira, M.C.; Arruda Neto, J.D.T. \& Cavalcanti, A.D.C. 2008. Temporal variation of the phytoplankton community at short sampling intervals in the Mundaú reservoir, Northeastern Brazil. Acta Botanica Brasilica 22(4): 970-982.

Figueredo, C.C. \& Giani, A. 2009. Phytoplankton community in the tropical lake of Lagoa Santa (Brazil): Conditions favoring a persistent bloom of Cylindrospermopsis raciborskii. Limnologica 39: 264-272.

Fiore, M.F.; Neilan, B.A.; Copp, J.N.; Rodrigues, J.L.M.; Tsai, S.M.; Lee, H. \& Trevors, J.T. 2005. Characterization of nitrogen-fixing cyanobacteria in the Brazilian Amazon floodplain. Water Research 39: 5017-5026.

Fonseca, B.M. \& Bicudo, C.E.M. 2008. Phytoplankton seasonal variation in a shallow stratified eutrophic reservoir (Garças Pond, Brazil). Hydrobiologia 600(1): 267-282.

Fonseca, B.M. \& Bicudo, C.E.M. 2010. How important can the presence/ absence of macrophytes be in determining phytoplankton strategies in two tropical shallow reservoirs with different trophic status? Journal of Plankton Research 32(1): 31-46.

Garcia, J.P.M.; Freitas, N.P. \& Filho, N.L.S. 1999. Caracterização geoambiental da Represa de Itupararanga. Disponível em: http://IPE. ibrc.unesp.br/cbhsmt/projeto.html (Acesso em 07/08/2009).

Gemelgo, M.C.P.; Mucci, J.L.N. \& Navas-Pereira, D. 2009. Population dynamics: seasonal variation of phytoplankton functional groups in Brazilian reservoirs (Billings and Guarapiranga, São Paulo). Brazilian Journal of Biology 69(4): 1001-1013.

Happey-Wood, C.M. 1988. Ecology of freshwater planktonic green algae. In: Sandgren, C.D. (Ed.). Growth and reproductive strategies of freshwater phytoplankton. Cambridge, Cambridge University Press.

Huszar, V.L.M. \& Caraco, N. 1998. The relationship between phytoplankton composition and physical-chemical variables: a comparison of 
taxonomic and morphological-functional descriptors in six temperate lakes. Freshwater Biology 40(4): 679-696.

Huszar, V.L.M. \& Reynolds, C.S. 1997. Phytoplankton periodicity and sequences of dominance in an Amazonian flood-plain lake (Lago Batata, Pará, Brasil): responses to gradual environmental change. Hydrobiologia 346(1-3): 169-181.

Huszar, V.L.M.; Silva, L.H.S.; Marinho, M.; Domingos, P. \& Sant'Anna, C.L. 2000. Cyanoprokaryote assemblages in eight productive tropical Brazilian waters. Hydrobiologia 424(1-3): 67-77.

INMET - Instituto Nacional de Meteorologia. 2009, 2010. Dados de monitoramento climático: temperatura do ar, precipitação pluviométrica e velocidade dos ventos. Disponível em: http://www. inmet.gov.br (Accesso em 07/06/2010).

Komarék, J. \& Fott, B. 1983. Chlorophyceae (Grünalgen). Ordiniung: Chlorococcales. In: HUBER-Pestalozzi, G. Das phytoplankton des süsswasers: systematic and biologie. E. Schwiezerbat'sche Verlagsbuch - handlung. Stutgart.

Lamparelli, M.C. 2004. Graus de trofia em corpos d'água do Estado de São Paulo: avaliação dos métodos de monitoramento. Tese de Doutorado. Instituto de Biociências, Universidade de São Paulo, São Paulo.

Lopes, M.R.M.; Bicudo, C.E.M. \& Ferragut, M.C. 2005. Short term spatial and temporal variation of phytoplankton in a shallow tropical oligotrophic reservoir, southeast Brazil. Hydrobiologia 542(1): 235-247.

Marciano, F.T. 2001. Estudo limnológico da bacia do rio Sorocaba (SP) e utilização do índice de integridade biótica da comunidade de peixes para avaliação ambiental. Dissertação de Mestrado. Escola de Engenharia de São Carlos, Universidade de São Paulo, São Carlos.

Melo, S. \& Huszar, V.L.M. 2000. Phytoplankton in an Amazonian floodplain lake (Lago Batata, Brasil): diel variation and species strategies. Journal of Plankton Research 22(1): 63-76.

Molisani, M.M.; Barroso, H.S.; Becker, H.; Moreira, M.O.P.; Hijo, C.A.G.; Monte, T.M. \& Vasconcellos, G.H. 2010. Trophic state, phytoplankton assemblages and limnological diagnosis of the Castanhão Reservoir, CE, Brazil. Acta Limnologica Brasiliensia 22(1): 1-12.

Moura, A.N.; Bittencourt-Oliveira, M.C.; Dantas, E.W. \& Arruda Neto, J.D.T. 2007. Phytoplanktonic associations: a tool to understanding dominance events in a tropical Brazilian reservoir. Acta Botanica Brasilica 21(3): 641-648.

Nabout, J.C.; Nogueira, I.S. \& Oliveira, L.G. 2006. Phytoplankton community of floodplain lakes of the Araguaia River, Brazil, in the rainy and dry seasons. Journal of Plankton Research 28(2): 181-193.

Padisák, J.; Crossetti, L.O. \& Naselli-Flores, L. 2009. Use and misuse in the application of the phytoplankton functional classification: a critical review with updates. Hydrobiologia 621(1): 1-19.

Padisák, J.; Molnár, G.; Soróczki-Pintér, E.; Hajnal, E. \& George, D.G. 2006. Four consecutive dry years in Lake Balaton (Hungary): consequences for phytoplankton biomass and composition. Verhandlungen des Internationalen Verein Limnologie 29: 1153-1159.

Palma, P.; Alvarenga, P.; Palma, V.L.; Fernandes, R.M.; Soares, A.M.V.M. \& Barbosa, I.R. 2010. Assessment of anthropogenic sources of water pollution using multivariate statistical techniques: a case study of the Alqueva's reservoir, Portugal. Environmental Monitoring and Assessment 165(1-4): 539-552.

Parra, O.O.; Gonzalez, M. \& Dellarossa, V. 1980. Manual taxonómico del fitoplancton de águas continentales com especial referencia al fitoplancton de Chile. Classe Euglenophyceae. Concepcion, Universidad de Concepcion.

Parra, O.O.; Gonzalez, M. \& Dellarossa, V. 1983. Manual taxonómico del fitoplancton de águas continentales com especial referencia al fitoplancton de Chile. Classe Chlorophyceae. Concepcion, Universidad de Concepcion.

Parra, O.O.; Gonzalez, M.; Dellarossa, V.; Rivera, P. \& Orellana, M. 1982. Manual taxonómico del fitoplancton de águas continentales com especial referencia al fitoplancton de Chile. Classe Cyanophyceae. Concepcion, Universidad de Concepcion.

Rangel, L.M.; Silva, L.H.S.; Arcifa, M.S. \& Perticarrari, A. 2009. Driving forces of the diel distribution of phytoplankton functional groups in a shallow tropical lake (Lake Monte Alegre, Southeast Brazil). Brazilian Journal of Biology 69(1): 75-85.

Reynolds, C.S. 1997. Vegetation processes in the pelagic: a model for ecosystem theory. Germany, Ecology Institute.

Reynolds, C.S.; Huszar, V.; Kruk, C.; Naselli-Flores, L. 2002. Towards a functional classification of the freshwater phytoplankton. Journal of Plankton Research 24(5): 417-428.

Salmaso, N. 2002. Ecological patterns of phytoplankton assemblages in Lake Garda: seasonal, spatial and historical features. Journal of Limnology 61(1): 95-115.

Salmaso, N. \& Padisák, J. 2007. Morpho-Functional Groups and phytoplankton development in two deep lakes (Lake Garda, Italy and Lake Stechlin, Germany). Hydrobiologia 578(1): 97-112.

Sant'Anna, C.L.; Azevedo, M.T.P., Agujaro, L.F., Carvalho, M.C.; Carvalho, L.R. \& Souza, R.C.R. 2006. Manual ilustrado para identificação e contagem de cianobactérias planctônicas continentais brasileiras. Rio de Janeiro, Interciência.

Sarmento, H. \& Descy, J.P. 2008. Use of marker pigments and functional groups for assessing the status of phytoplankton assemblages in lakes. Journal of Applied Phycology 20(6): 1011-1011.

Silva, C.A.; Train, S. \& Rodrigues, L.C. 2005. Phytoplankton assemblages in a Brazilian subtropical cascading reservoir system. Hydrobiologia 537(1-3): 99-109.

Sarmento, H.; Isumbisho, M. \& Descy, J.P. 2006. Phytoplankton ecology of Lake Kivu (eastern Africa). Journal of Plankton Research 28(9): 815-829.

Soylu, E.N. \& Gönülol, A. 2010. Functional Classification and Composition of Phytoplankton in Liman Lake. Turkish Journal of Fisheries and Aquatic Sciences 10: 53-60.

Wilhelm, S. \& Adrian, R. 2008. Impact of summer warming on the thermal characteristics of a polymictic lake and consequences for oxygen, nutrients and phytoplankton. Freshwater Biology 53(2): 226-237.

Willén, E. 2003. Dominance patterns of planktonic algae in Swedish forest lakes. Hydrobiologia 502(1-3): 315-324.

Zhang, X.; Xie, P.; Chen, F.; Li, S. \& Qin, J. 2007. Driving forces shaping phytoplankton assemblages in two subtropical plateau lakes with contrasting trophic status. Freshwater Biology 52(8): 1463-1475. 